\title{
Safety and Competency are the Main Priorities in Pediatric Endoscopy
}

\author{
Byung-Ho Choe \\ Department of Pediatrics, School of Medicine, Kyungpook National University, Daegu, Korea
}

See "Endoscopic Yield, Appropriateness, and Complications of Pediatric Upper Gastrointestinal Endoscopy in an Adult Suite: A Retrospective Study of 822 Children" by Manzoor Ahmad Wani, Showkat Ali Zargar, Ghulam Nabi Yatoo, et al., on page $436-442$.

Safety is always the first priority in pediatric endoscopy. Quality control is crucial to prevent cardiopulmonary adverse events related to endoscopy and/or sedation, bleeding, infection, and perforation. ${ }^{1}$ One cannot emphasize enough on the importance of safety in children, particularly if the procedure is performed in an adult unit. Risk evaluation by the pediatrician is necessary before sedation. Moreover, sedation- and anesthesia-related events should be minimized. The sedation strategy should be applied based on the safety and effectiveness. The optimal choice among several agents may differ across endoscopic centers. ${ }^{2}$ A prompt response to adverse events by a trained pediatrician and/or an anesthesiologist is vital.

On-site monitoring devices, including the pulse oximeter and electrocardiogram monitor, suction equipment, oxygen supply, and emergency cart, should be checked. ${ }^{3}$ When conducting an effective and rapid endoscopic procedure to minimize adverse events, pediatric endoscopes as well as smaller accessories (e.g., forceps, injection needle, retrieving net, snare, etc.) should be available in consideration of age and

\footnotetext{
Received: May 6, 2020 Revised: May 26, 2020

Accepted: June 4, 2020

Correspondence: Byung-Ho Choe

Department of Pediatrics, School of Medicine, Kyungpook National University, 130 Dongdeok-ro, Jung-gu, Daegu 41944, Korea

Tel: +82-53-200-5704, Fax: +82-53-425-6683, E-mail: bhchoe@knu.ac.kr ORCID: https://orcid.org/0000-0001-9899-9120
}

(c) This is an Open Access article distributed under the terms of the Creative Commons Attribution Non-Commercial License (http://creativecommons.org/ licenses/by-nc/3.0) which permits unrestricted non-commercial use, distribution, and reproduction in any medium, provided the original work is properly cited. body weight. However, standard endoscopy is often preferred in special situations, such as therapeutic endoscopic procedures. ${ }^{4,5}$ In emergency situations, even endoscopy used for the toddler age group can be used for newborn infants.

In this issue of Clinical Endoscopy, Wani et al. demonstrated that pediatric endoscopy for children over 4 months of age was safely performed by adult gastroenterologists in conjunction with a pediatrician. ${ }^{6}$ This finding is supportable in most of the countries where pediatric endoscopists are few. Furthermore, acknowledging the presence of a pediatric gastroenterologist, the collaboration of an adult gastroenterologist is vital for the optimal management of pediatric patients because adult endoscopists are capable of performing more complex endoscopic procedures (e.g., endoscopic retrograde cholangiopancreatography, endoscopic ultrasonography, and stent placement). Authors from Kashmir, India, had reported 110 pediatric cases of therapeutic endoscopy (e.g., active bleeding control, variceal ligation, foreign body removal, etc.). ${ }^{6}$

To comment from the standpoint of a pediatric gastroenterologist, I have some tips based on my experience and the Internet. Endoscopic variceal ligation (EVL) is often required for infants with advanced liver cirrhosis because of biliary atresia awaiting liver transplantation. When an outer cap is attached to the distal end of the endoscope and then inserted into the esophagus of the infant, sudden desaturation may develop because of narrowing of the airway due to compression of the posterior wall of the trachea by the cap. Based on the Poiseuille equation, the flow is inversely proportional to the radius to the fourth power. As an EVL cap is not available for a smaller endoscope, sclerotherapy using a pediatric injection needle may 
be considered. For removing esophageal or gastroduodenal foreign bodies in children (generally infants), preprocedural simulation is necessary to achieve safe and rapid removal. Disk battery impaction in the esophagus is a real emergency case. ${ }^{5}$ Removing foreign bodies using Foley catheters or magnet-attached Levine tubes could be an option in real emergencies when immediate therapeutic endoscopy is not possible.

There have been paradigm shifts in pediatric gastrointestinal diseases in the 21st century. The prevalence rates of eosinophilic gastroenteritis (and esophagitis) and inflammatory bowel disease have been rapidly increasing among East Asian children in the recent decade. ${ }^{8,9}$ An index of suspicion is essential for an early diagnosis. Even when the gross endoscopic appearance is normal or nonspecific, it is necessary to perform a biopsy and verify with the pathologist to obtain eosinophil counts for diagnosing eosinophilic gastroenteritis. ${ }^{8}$

In addition, based on the gross appearance of the colonic mucosa, there are several diseases mimicking ulcerative colitis, such as infectious enterocolitis caused by Salmonella, Shigella, Campylobacter, Yersinia, and Entamoeba. For screening newly emerging enteropathogens, such as Campylobacter and Yersin$i a$, in East Asian countries, real-time multiplex polymerase chain reaction is required, as the conventional culture results are generally negative because of the requirements of selective media and special incubation conditions. ${ }^{10}$ For achieving the best efficiency of pediatric endoscopy beyond avoiding unnecessary reinspection, adult gastroenterologists should be aware of the patient's information of the disease and condition.

Regarding infectious diseases, such as those caused by Shigella, Salmonella, and Entamoeba, the prevalence of Helicobacter pylori infection is also rapidly decreasing in Korean children and is presumed to be $<5 \%$. Nevertheless, $H$. pylori should be screened in endemic areas because gastrointestinal bleeding can be fatal for children with bleeding disorders.

In conclusion, it would be ideal to have adequate children's hospitals worldwide, and a pediatric gastroenterologist should be able to perform difficult endoscopic procedures with the cooperation of an adult gastroenterologist and an anesthesiologist; however, in reality, there are many countries where this is not possible. It may be more dangerous to transport such pa- tients to bigger centers in lieu of all conditions, particularly in case of a pediatric emergency. In this regard, it is essential that the adult gastroenterologist collaborates with a pediatrician. Wani et al. provided meaningful data pertaining to pediatric diagnostic and therapeutic endoscopy. ${ }^{6}$ It is important for both pediatric and nonpediatric endoscopists to be familiar with the pediatric guidelines that have already been developed. ${ }^{1,5}$

Conflicts of Interest

The author has no financial conflicts of interest.

\section{REFERENCES}

1. Lightdale JR, Liu QY, Sahn B, Troendle DM, Thomson M, Fishman DS. Pediatric endoscopy and high-risk patients: a clinical report from the NASPGHAN endoscopy committee. J Pediatr Gastroenterol Nutr 2019;68:595-606.

2. Oh SH. Sedation in pediatric esophagogastroduodenoscopy. Clin Endosc 2018;51:120-128.

3. Coté CJ, Wilson S. Guidelines for monitoring and management of pediatric patients before, during, and after sedation for diagnostic and therapeutic procedures. Pediatrics 2019;143:e20191000.

4. Schreiber-Dietrich D, Hocke M, Braden B, Carrara S, Gottschalk U, Dietrich CF. Pediatric endoscopy, update 2020. Appl Sci (Basel) 2019;9:5036.

5. Tringali A, Thomson M, Dumonceau JM, et al. Pediatric gastrointestinal endoscopy: European Society of Gastrointestinal Endoscopy (ESGE) and European Society for Paediatric Gastroenterology Hepatology and Nutrition (ESPGHAN) guideline executive summary. Endoscopy 2017;49:83-91.

6. Wani MA, Zargar SA, Yatoo GN, et al. Endoscopic yield, appropriateness, and complications of pediatric upper gastrointestinal endoscopy in an adult suite: a retrospective study of 822 children. Clin Endosc 2020;53:436-442.

7. Choe JY, Choe BH. Foreign body removal in children using foley catheter or magnet tube from gastrointestinal tract. Pediatr Gastroenterol Hepatol Nutr 2019;22:132-141.

8. Choi BS, Hong SJ, Park SH, Kim HM, Choe BH. Differences in features and course of mucosal type eosinophilic gastroenteritis between Korean infants and children. J Korean Med Sci 2015;30:1129-1135.

9. Kang B, Kim JE, Jung JH, et al. Korean children and adolescents with Crohn's disease are more likely to present with perianal fistulizing disease at diagnosis compared to their European counterparts. Pediatr Gastroenterol Hepatol Nutr 2020;23:49-62.

10. Valledor S, Valledor I, Gil-Rodríguez MC, Seral C, Castillo J. Comparison of several real-time PCR kits versus a culture-dependent algorithm to identify enteropathogens in stool samples. Sci Rep 2020;10:4301. 